\title{
Theoretical and practical aspects of the waste water electrochemical purification method's applicability
}

\author{
Darya Ignatkina $^{1 *}$, Irina Shevtsova ${ }^{1}$, Victoria Telyatnikova ${ }^{2}$, and Daria Kotlomina ${ }^{1}$ \\ ${ }^{1}$ Volgograd State Technical University (VolgSTU), 400074, Volgograd, Russia \\ ${ }^{2}$ Volgograd State Social and Pedagogical University (VSSPU), 400005, Volgograd, Russia
}

\begin{abstract}
In this article, the authors highlight one of the important directions in the field of environmental protection, namely, an increase in the amount of waste in the form of wastewater (WW) entering water bodies, most often without the required degree of purification. The main reason for the indicated problem development is the rapid growth of industrial production. The authors of the article carried out a literary search for effective and economically justified methods of industrial wastewater treatment. The article presents the results of laboratory studies proving the industrial wastewater electrochemical treatment efficiency and at the same time provides a theoretical explanation of the obtained experimental data from a physical, mathematical and chemical point of view, which allows not only to assess the choice reliability of juice processing some optimal parameters on the example of a food industry, but also take into account the design features in the development of engineering and technical devices, in particular - the electrolyzers.
\end{abstract}

\section{Introduction}

The discharge of untreated wastewater (WW) into water bodies remains a serious problem in modern conditions. Percentage of regulatory cleared WW to the volume of wastewater requiring treatment is $14.9 \%$ in industry, in housing and communal services is $7.8 \%$, and in agriculture $-0.6 \%[1]$.

In the food industry, about 60 million are used annually $\mathrm{m}^{3}$ water, the volume of discharges is 46 million $\mathrm{m}^{3}$. Share of contaminated WW to the total volume of water reaches $77 \%$, which indicates the low efficiency of the existing treatment facilities, or their absence [2].

Today, in the food industry, in particular at the enterprises of the tobacco industry of the Russian Federation, cleaning WW most often based on physical and chemical methods, which are used both independently and in combination with mechanical methods $[2,3]$.

It should be noted that physical and chemical methods are quite versatile, technologically and economically tested, capable of ensuring stable operation of structures at low liquid temperatures, as well as $\mathrm{pH}$, when toxic substances enter [3-5]. The

\footnotetext{
*Corresponding author: viv_vgasu@mail.ru
} 
considered group of methods includes such cleaning methods WW, as adsorption, flotation, reverse osmosis, coagulation, extraction, electrocoagulation, electro flotation, electrodialysis, etc. One of the undoubted advantages of these methods is that a much shorter processing time for WW is required.

Of the electrochemical methods, the most widely recognized methods are electrochemical coagulation (electrocoagulation), electrochemical flotation (electro flotation) and electrochemical oxidation. The wastewater treatment effect is as follows: suspended solids - $90-95 \%$, surfactants - $60-70 \%$, fats - $75-95 \%$, oil products - up to $95 \%$, chromium - $90-98 \%[3,4,6]$.

\section{Main part}

This article provides a theoretical explanation of the experimental data obtained, indicating the applicability of the electrochemical oxidation method for cleaning WW enterprises of the tobacco industry in the Russian Federation. Experts in the field of water supply and sanitation consider it to be quite promising, the most environmentally friendly and effective $[1,4,7]$. The essence of the electrochemical oxidation method is that water passes through a container of a special design, in which complex redox reactions occur under the constant electric current influence.

At present, a huge amount of experimental material has been accumulated on the existence of electro organic reactions $[8,9]$. However, their implementation in practice is constrained by insufficient knowledge of the mechanism and redox processes kinetics. In addition, the oxidizing and reducing agents obtained during electrolysis have a particularly large reserve of chemical energy at the moment of their formation, which significantly complicates the course of the organic compounds' electrosynthesis, since it can be difficult to direct the process towards obtaining the main commercial product or to stop the redox transformation reactions at the desired stage. $[9,10]$. In this case, the products of deeper oxidation or even destructive decomposition can be obtained as a result of the main carbon - carbon chain sufficiently strong bonds' destruction, up to carbon dioxide and water formation [11].

When cleaning WW - this circumstance, on the contrary, is very positive, since the main tasks of neutralizing industrial wastewater include the need for the destruction of polluting components to simpler and more harmless products [12].

The authors conducted the laboratory studies to study the process of electrochemical purification (electrooxidation)WW enterprises of the tobacco industry in the Russian Federation, the composition of which is presented in Table 1.

Table 1. Structure WW enterprises of the tobacco industry in the Russian Federation

\begin{tabular}{|l|c|}
\hline \multicolumn{1}{|c|}{ Contamination name } & Concentration, $\mathrm{mg} / \mathrm{dm}^{3}$ \\
\hline Suspended substances & 181 \\
\hline BOD full & 1856 \\
\hline COD & 2830 \\
\hline Iron gen. & 0.2 \\
\hline Fats & 5.9 \\
\hline Ammonium (NH4) & 32.5 \\
\hline Petroleum products & 0.94 \\
\hline Anonic surfactants & 21.8 \\
\hline Phosphates & 0.56 \\
\hline Chlorides & 59 \\
\hline Sulfates & 75 \\
\hline
\end{tabular}




\begin{tabular}{|l|c|}
\hline $\mathrm{pH}$ & 6.69 \\
\hline
\end{tabular}

The purification process consisted in passing the waste liquid into the interelectrode space of the electrolyzer, on the electrodes of which a potential difference of 3-5 V. It should be taken into account that such phenomena as water electrolysis, particle polarization, electrophoresis, redox processes could occur in the electrolyzer, interaction of electrolysis processes with each other $[9,12]$, these processes from a physical, mathematical and chemical point of view can be explained as follows:

1) when heated or frozen, when electrolytes are added to water, when a magnetic field is applied, the aggregate stability of impurities is violated. Suspended water can contain hydrophilic and hydrophobic impurities. Hydrophilic ones are mainly represented by organic substances, while hydrophobic ones are represented by sulfates, phosphates, metal impurities, clay particles, dusty grains of sand, etc. A significant amount of water is retained on the surface of hydrophilic impurities in the form of a hydration shell, due to the presence of surface polar groups, such as $-\mathrm{OH}-,-\mathrm{COOH}-$ and others. They also keep the hydration shell around the particle. Being in thermal Brownian motion, hydrophilic particles diffuse together with the hydration shell, usually carry small electric charges and, as a rule, do not coagulate under the influence of electrolytes. Hydrophobic impurities are almost devoid of hydration shells, but they have an electric double layer and carry significant electric charges. Usually, hydrophobic particles have a crystalline structure and a large specific surface area on which ions present in water are adsorbed. It is characteristic that all particles of a given substance collect mainly ions of the same sign, which reduce the free surface of the energy of hydrophobic particles. The ions are adjacent directly to the nucleus form a surface-nuclear layer (adsorption layer). Since an electric charge is formed at the adsorption layer boundary, a diffusion layer of oppositely charged ions is created around the core with the adsorption layer (granule), which compensates for the granule charge. At rest, such impurity particles are electrically neutral, since the charge of the granule is neutralized by oppositely charged ions of the diffuse layer. Being in a state of Brownian motion, the ions of the diffuse layer of the impurity particle lag behind, detach from the granule, and the particle acquires an electric charge. Thus, particles with the same charges, when interacting, repel each other, and the particles with opposite charges are attracted;

2) Along with the processes described above, it should be noted that between the colloidal impurities of water there are molecular forces of mutual attraction, called van der Waals forces, which act at a small distance between interacting particles and decrease with increasing distance between them, and when particles approach, the repulsive forces and the attraction increases. At the same time, initially the repulsive force prevails over the gravity force. However, if the particles, moving with high speed, overcome the specified force barrier, then the forces of attraction become prevailing and such particles stick together and grow larger. The force barrier magnitude is characterized by the electrokinetic potential. It is optimal when the electrokinetic potential is zero, and since most colloidal particles WW have a negative charge in the granule, it becomes necessary to neutralize it, which can be achieved by additional introduction of electrolyte.

The main part of the research carried out to determine the optimal values of the factors affecting the conduct of the electrochemical oxidation process WW enterprises of the tobacco industry in the Russian Federation, allow us to assert that the following parameters must be taken into account for the effective treatment of waste liquid:

1) selection of anode material and certain operating parameters of processing: current density, treatment time of effluents, as well as temperature, $\mathrm{pH}$ of the medium and concentration of sodium chloride solution [12]. Therefore, further studies were devoted to the study of the kinetics of reactions occurring in the near-electrode spaces. The results 
obtained showed that destruction of organic substances occurs in the anode space, which determine the values $\mathrm{BOD}_{\text {full }}$ and COD considered WW and only a small fraction of them polymerize at the cathode. Based on this, it can be concluded that electrochemical neutralization WW It is more expedient to carry out the oxidation of organic compounds at the anode, the choice of the material of which should be given special attention, since one of the main problems is that most metals are thermodynamically unstable under conditions of anodic polarization [10,11]. The most suitable materials for electrodes are metals with low overvoltage, such as platinum. However, due to economic factors, according to sources [11], in devices (electrolyzers) for electrooxidation of polluting components WW insoluble metal oxide anodes - ruthenium-titanium oxide anode, cobalt oxide titanium anode, titanium manganese dioxide anode (titanium base with an active surface coating with a mixture of titanium, ruthenium, manganese and cobalt oxides, respectively) as well as graphite electrodes are used. When choosing the anode material, the studies were carried out on real WW composition, which is presented in table 1 at current density $-3,7 \mathrm{~A} / \mathrm{dm}^{2}$ and a processing time of 20 minutes. As a result, the following dependences of the cleaning efficiency from the anode material were obtained (Fig. 1).

Thus, according to the experiments results, the following conclusions can be drawn: the maximum cleaning efficiency with the shortest processing time (15 minutes) and current density $3,5 \mathrm{~A} / \mathrm{dm}^{2}$ are achieved by carrying out the electrooxidation process on the cobalt oxide titanium anode electrodes. However, the use of cobalt oxide titanium anode in industrial conditions leads to a significant increase in the cost of the cleaning process, therefore it is advisable to use it as ruthenium-titanium oxide anode, important cleaning efficiency close to cobalt oxide titanium anode. The use of graphite as an anode material does not allow achieving purification efficiency values close to titanium with an active coating based on ruthenium dioxide and cobalt oxide.;

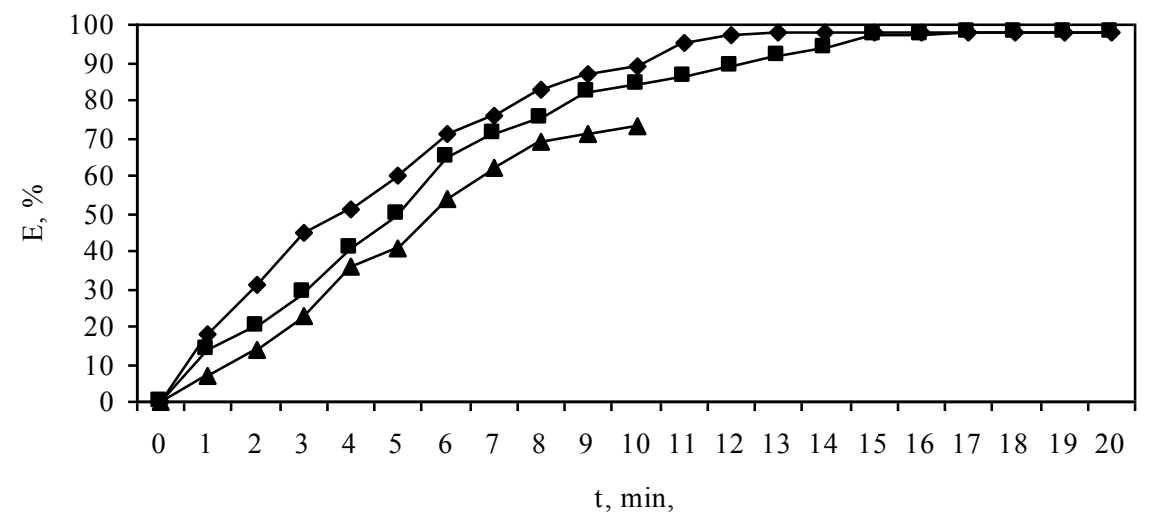

$\multimap$ Graph $1 \rightarrow-$ Graph $2 \multimap-$ Graph 3

Fig. 1. Dependence of the cleaning efficiency from the anode material (WW enterprises of the tobacco industry in the Russian Federation, current density $3,7 \mathrm{~A} / \mathrm{dm}^{2}$, processing time 20 minutes): 1 - cobalt oxide titanium anode; 2 - ruthenium-titanium oxide anode; 3 - graph.

2) the need to add an aqueous solution $\mathrm{NaCl}$ and the choice of its optimal concentration, the results of this study are presented in Fig. 2. 


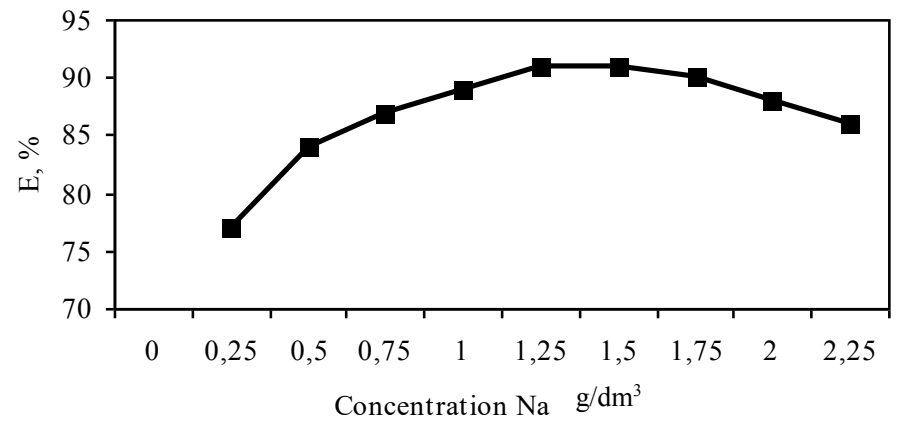

Fig. 2. Dependence of cleaning efficiency WW enterprises of the tobacco industry of the Russian Federation from concentration $\mathrm{NaCl}$ (anode - ruthenium-titanium oxide anode, current density 3,5 $\mathrm{A} / \mathrm{dm}^{2}$, processing time - 15 minutes, solution temperature $20^{\circ} \mathrm{C}, \mathrm{pH}$ solution medium -7).

The results obtained in Fig. 2, may indicate that the yield of electrolysis products of the solution $\mathrm{NaCl}$ influences the selectivity of electrochemical reactions on the electrodes and chemical reactions in the volume of the electrolyte. When a direct electric current passes through an electrolyte containing ions $\mathrm{Na}^{+}, \mathrm{Cl}^{-}, \mathrm{H}^{+}$and $\mathrm{OH}^{-}$, the sequence of the discharge of these ions at the electrodes is initially determined by the values of their standard electrode potentials. At the cathode, exclusively hydrogen evolution occurs, since the sodium discharge potential is high. The standard chlorine potential is more electrically positive than $\mathrm{OH}^{-}$ions, but on oxide-ruthenium anodes $\mathrm{OH}^{-}$ions are discharged with a large overvoltage, therefore the main anodic process is the release of chlorine.

The chemical reactions that take place in brine during the electrooxidation process can be described as follows:

$$
2 \mathrm{NaCl} \rightarrow 2 \mathrm{Na}^{+}+2 \mathrm{Cl}^{-}
$$

Cathode reaction:

$$
2 \mathrm{Na}^{+}+2 e \rightarrow 2 \mathrm{Na} ; 2 \mathrm{Na}+2 \mathrm{H}_{2} \mathrm{O} \rightarrow 2 \mathrm{NaOH}+\mathrm{H}_{2} \uparrow
$$

Reaction at the anode:

$$
2 \mathrm{Cl}^{-} \rightarrow 2 \mathrm{Cl}+2 \mathrm{e} ; \mathrm{Cl}+\mathrm{Cl} \rightarrow \mathrm{Cl}_{2} \uparrow
$$

The total reaction in the electrolytic cell can be expressed by the equation:

$$
2 \mathrm{NaCl}+2 \mathrm{H}_{2} \mathrm{O} \rightarrow \mathrm{Cl}_{2}+2 \mathrm{NaOH}+\mathrm{H}_{2} \uparrow ;
$$

3) the accumulation of a large number of gas bubbles on the electrode plates' surface, which was recorded in laboratory studies of the real WW enterprises electrooxidation processes of the tobacco industry in the Russian Federation, which is most likely explained by the formation of chlorine bubbles at the anode and hydrogen at the cathode, which floating on their surface suspended and colloidal particles of pollution.

The diameter of hydrogen bubbles is from 20 to 100 microns. Small bubbles help improve the efficiency of the electro flotation process. However, to obtain bubbles of the required size, it is necessary to choose the right material, shape, cathode wire diameter, and current density [13].

The dependence of the number and size of the formed hydrogen bubbles on the electrode surface wire cathodes and its curvature was studied by B.M. Matov [14].

Let us consider the behavior of a gas bubble as it rises through the water surface. We will assume that a hydrogen bubble has formed on the cathode near the bottom of the purification chamber (we take the bubble diameter equal to $50 \mu \mathrm{m}$ ) and evenly rises to the 
surface through a layer of water $0.5 \mathrm{~m}$ thick. At the initial moment of time (near the bottom), the pressure inside the bubble $P_{1}$ is defined by the formula (1):

$$
P_{1}=P_{a}+P_{h y d r}+P_{l a p l 1}
$$

where $P_{a}=10^{5} \mathrm{~Pa}$ defines atmosphere pressure;

$P_{h y d r}=\rho g h(2)$ is hydrostatic fluid pressure;

$P_{\text {lapl1 }}=\frac{\sigma}{2 R_{1}}$ (3) is Laplace pressure $\left(\sigma=72 \cdot 10^{-3} \mathrm{~N} / \mathrm{m}\right.$ is the coefficient of surface tension of water under normal conditions, $R_{1}$ is bubble radius near the bottom).

At the final moment of time (near the surface of the liquid) the pressure inside the bubble $P_{2}$ is defined by the formula (2):

$$
P_{2}=P_{a}+P_{\text {lapl } 2},
$$

where $P_{\text {lapl } 2}=\frac{\sigma}{2 R_{2}}, R_{2}$ is a bubble radius near surface.

We will assume that the temperature of the liquid in the volume of the cleaning chamber is constant, therefore, for a bubble in the liquid, the Boyle-Mariotte law must be fulfilled, that is $P_{1} V_{1}=P_{2} V_{2}$. Where $P_{1}, V_{1}$ define pressure and volume of the bubble near the bottom, and $P_{2}, V_{2}$ define pressure and volume of the bubble near the water surface. By changing the pressure $P_{1}$ and $P_{2}$ it is possible to judge the change in the volume of the bubble when it moves through the water column.

Let us estimate the quantities included in the formula (1):

$$
\begin{gathered}
P_{h y d r}=\rho g h=1000 \cdot 10 \cdot 0,5=5 \cdot 10^{3} \mathrm{~Pa} \\
P_{\text {lapl } 1}=\frac{\sigma}{2 R_{1}}=\frac{72 \cdot 10^{-3}}{50 \cdot 10^{-6}}=1,44 \cdot 10^{3} \mathrm{~Pa}
\end{gathered}
$$

From the above-shown calculations, it can be concluded that the pressure values:

$$
\begin{gathered}
P_{1}=10^{5}+5 \cdot 10^{3}+1.44 \cdot 10^{3}=1.0644 \cdot 10^{5} \mathrm{~Pa} \\
\text { and } \\
P_{2}=10^{5}+1.44 \cdot 10^{3}=1.0144 \cdot 10^{5} \mathrm{~Pa}
\end{gathered}
$$

differ little from each other (by about $5 \%$ ).

Now let us estimate how the volume of the bubble changes in this case.

$$
\begin{gathered}
P_{1} V_{1}=P_{2} V_{2}, \Rightarrow V_{2}=\frac{P_{1} V_{1}}{P_{2}}, \Rightarrow \frac{4 \pi}{3} R_{2}^{3}=\frac{P_{1}}{P_{2}} \frac{4 \pi}{3} R_{1}^{3}, R_{2}=\sqrt[3]{\frac{P_{1}}{P_{2}}} \cdot R_{1}, \Rightarrow \\
R_{2}=\sqrt[3]{\frac{P_{1}}{P_{2}}} \cdot R_{1}=\sqrt[3]{\frac{1,0644 \cdot 10^{5}}{1,0144 \cdot 10^{5}}} \cdot 25 \cdot 10^{-6}=25.4 \cdot 10^{-6} \mathrm{~m}
\end{gathered}
$$

\section{Conclusion}


Based on the above-performed mathematical calculations, it can be argued that during the gas bubble movement through water, its radius practically does not change, and therefore its floating properties do not change.

Thus, the above-made theoretical substantiation of the WW electrochemical cleaning process enterprises of the tobacco industry in the Russian Federation from a physical, mathematical and chemical point of view, made it possible to assess the reliability of the choice of some optimal processing parameters for the considered effluents obtained experimentally, which in the future can serve as the basis for determining the technological mode of removing polluting components' operation depending on the quality of the incoming water, as well as taking into account the design features in the engineering and technical devices' development, in particular - electrolyzers:

1) Metals with low overvoltage are most suitable as electrode materials. According to the results of the experiments carried out, it can be argued that the maximum cleaning efficiency with the shortest processing time (15 minutes) and current density 3,5 A/ $\mathrm{dm}^{2}$ achieved by carrying out the electrooxidation process on the electrodes cobalt oxide titanium anode;

2) When adding an aqueous solution $\mathrm{NaCl}$ exclusively hydrogen evolution occurs at the cathode, since the sodium discharge potential is high. The standard chlorine potential is more electrically positive than $\mathrm{OH}^{-}$ions, but on oxide-ruthenium anodes $\mathrm{OH}^{-}$ions are discharged with a large overvoltage, therefore the main anodic process is the release of chlorine;

3) The presented mathematical calculations, describing the dependences of the accumulation of a large number of gas bubbles on the electrode plates' surface showed that during the gas bubble movement through water, its radius practically does not change, and therefore its floating properties do not change.

\section{References}

1. N.P. Shapkin, Physical and chemical studies of wastewater treatment [Text], Weystek2003: materials of the international. Congress - Moscow, 2003.

2. L.I. Arkhipov, V.A. Gorbenko, A.L. Efrimov, Processes and apparatuses of technical water supply systems of industrial enterprises. Textbook on the course "Energy use in energy and technology", Moscow.

3. L.V. Gandurina, E.V. Fomicheva, Water supply and sanitary engineering 5, 17-20 (2004).

4. G. Taralas M.G. Kontominas, Energy \& Fuels 19 (3), 1179-1185 (2005).

5. M. Bressan, Journal of Agricultural \& Food Chemistry 52 (5), 1228-1233 (2004).

6. S.A. Mayorov, Yu.A. Sedov, Yu.A. Parakhin, Environmental safety in the Technosphere 2010: materials of All-Russian scientific and technical internet conference - Eagle, 2011.

7. D.O. Ignatkina, Water supply and sanitary equipment 3, 36-44 (2018).

8. D.O. Ignatkina, Pat. 178983 Russian Federation, C02F 9/06 (2006.01). Device for wastewater treatment from multicomponent pollution, VolgSTU. No. 2017139298 ; statement 13.11.2017; publ. 24.04.2018, 12.

9. I.G. Krasnoborodko, S.V. Yakovlev, Technology of electrochemical water purification (Stroyizdat, Leningrad, 1987).

10. S.V. Fursov, T.O. Akhmetov, Electrochemical methods of wastewater treatment, Lowwaste, resource-saving chemical technologies and environmental safety: materials of 
All-Russian scientific and technical internet conference, Sterlitamak, Printing house "Phobos", 2013.

11. J. Gordon, Organic chemistry of electrolyte solutions (Mir, Moscow, 1979).

12. S.V. Yakovlev, I.G. Krasnoborodko, V.M. Rogov, Technology of electrochemical water purification (Stroyizdat, Leningrad, 1987).

13. A.L. Rotinyan, K.L. Tikhonov, I.A. Shoshina, Theoretical electrochemistry (Chemistry, Leningrad, 1981).

14. B.M. Matov, Electro flotation wastewater treatment (Kartya Moldoveniasca, Chisinau, 1982). 